\title{
An Ohmic heating non-local diffusion-convection problem for the Heaviside function
}

\author{
N.I. Kavallaris* $\quad$ D.E. Tzanetis ${ }^{\dagger}$ \\ (Received 4 June 2001; revised 23 May 2002)
}

\section{Abstract}

We study the non-local equation

$$
u_{t}=u_{x x}-u_{x}+\frac{\lambda f(u)}{\left(\int_{0}^{1} f(u) d x\right)^{2}}
$$

together with some initial and boundary conditions for the case where $f$ is the Heaviside function. It is found, for decreasing $f$, that there exist critical values $\lambda_{*}$ and $\lambda^{*}$, so that for $0<\lambda<\lambda_{*}$ there is a unique steady state solution which is asymptotically stable and the solution $u$ is global in time. For $\lambda_{*}<\lambda<\lambda^{*}$ there exist two steady-states and we study

${ }^{*}$ Department of Mathematics, Faculty of Applied Sciences, National Technical University of Athens, Zografou Campus, 15780 Athens, Greece. mailto:nkaval@math.ntua.gr

${ }^{\dagger}$ as above, mailto:dtzan@math.ntua.gr

${ }^{0}$ See http://anziamj . austms .org. au/V44/E030 for this article, (C) Austral. Mathematical Soc. 2002. Published September 19, 2002. ISSN 1446-8735 
their stability, while for $\lambda>\lambda^{*}$ there is no steady-state. Also, it is proved that for $\lambda>\lambda^{*}$ or for $\lambda_{*}<\lambda<\lambda^{*}$ and initial data sufficiently large, the solution $u$ "blows up" (in some sense). Moreover, for increasing $f$ and Neumann boundary conditions, $u$ is an unbounded solution global in time.

\section{Contents}

1 Introduction

E115

2 Existence and uniqueness

E118

3 Asymptotic behaviour and "blow-up"

E121

3.1 The mixed problem . . . . . . . . . . . E121

3.1.1 Stability . . . . . . . . . . . . . E E124

3.1.2 "Blow-up" for $\lambda>\lambda^{*}=e \ldots \ldots$. . . . E128

3.2 The Dirichlet problem . . . . . . . . . . . . E129

3.2.1 Stability . . . . . . . . . . . . . E133

3.2.2 "Blow-up" for $\lambda>\lambda^{*} \ldots \ldots \ldots \ldots$. . . E137

4 Conclusions

E139

References

E140

\section{Introduction}

One of the important aspects in food processing is heating and sterilization. A variety of methods are available, which aim in heating the food material to a temperature high enough so that food is adequately sterilized. An increasing in use method, which also permits 
to heat the food quickly and evenly so that the quality of food remains high (the food is not overcooked) is Ohmic heating. This method was originally developed at ERDC, Capenhurst, England and its physical behaviour is reported in $[2,15,17]$. More precisely, the food is passed through a conduit, part of which lies between two electrodes. A high electric current flowing between the electrodes results, in Ohmic heating of the food which quickly gets hot.

A mathematical model which describes such a process, in the case where heat convection dominates heat conduction, was considered by Please, Schwendeman and Hagan [14]. This model consists of a system of partial differential equations. They also studied the stability of models allowing for different types of flow. Both homogeneous and inhomogeneous cases were examined. (More background on this type of process can be found in $[2,7,8,15,17,20]$.)

Lacey, Tzanetis and Vlamos [12] considered the basic one-dimensional model, again when heat convection dominates heat conduction, for the simplest case of a plug flow of a homogeneous material and succeeded in reducing the system examined in [14] to a simple but non-local hyperbolic equation. Also in [12] was examined the stability and blow-up of the solutions of the problem, with respect to the value of the potential difference applied at the electrodes and for a variety of monotonic (with respect to the temperature) electrical resistivities.

In [9] we considered velocities for the food so that heat convection and heat conduction coexist and modelling closely parallels that of [12] we arrived at the non-local parabolic equation:

$$
u_{t}=u_{x x}-u_{x}+\frac{\lambda f(u)}{\left(\int_{0}^{1} f(u) d x\right)^{2}}, \quad 0<x<1, \quad t>0,
$$

where $u=u(x, t)$ represents the dimensionless temperature of the 
food, $\lambda=a V^{2}, a>0$ ( $a$ is a constant, $V$ is the potential difference) and $f(u)$ is the temperature-dependent electrical resistivity of the food. The previous equation has arisen after scaling the distance with the length of the channel, which makes the convective velocity one. Depending upon the substance undergoing the heating, the resistivity may be an increasing, a decreasing, or a non-monotonic function of temperature. For most foods conductivity increases with temperature so $f(u)$ is decreasing. Note that if one were to consider an alternative processing technique, one in which there was a flow in a channel whose walls were the electrodes and across which a prescribed current flowed, a very similar model would apply. This model would differ, however, in that conductivity would replace resistivity $f$. For this reason in [9] we examined the behaviour of solutions of equation (1) where $f$ is a strictly positive, Lipschitz continuous and monotonic function.

In this work, we study equation (1) together with some initial and boundary conditions for the case of Heaviside function, emphasizing in the case where

$$
f(s)=H(1-s), \quad s \geq 0 ;
$$

we also make a comment for $f(s)=H(s-1), s \geq 0$. Here $H$ denotes the Heaviside function:

$$
H(s)= \begin{cases}0, & s<0, \\ 1, & s \geq 0 .\end{cases}
$$

The particular functional form of $f(u)$ is determined by the physical problem being considered, since many food materials have resistivities that vary considerably during the process. The same happens with ceramic materials in thermistors [3, 4, 5, 10]. In general, the Heaviside function is a good approximation for a number of physical quantities $[16,19]$. From the mathematical point of view, the 
Heaviside function is neither Lipschitz nor strictly positive, and so the techniques used in [9] should be modified.

Here we focus our attention on the problem

$$
\begin{aligned}
& u_{t}-u_{x x}+u_{x}=\frac{\lambda H(1-u)}{\left(\int_{0}^{1} H(1-u) d x\right)^{2}}, \quad 0<x<1, t>0,(3 \mathrm{a}) \\
& \mathcal{B}(u(x, t))=0, \quad x=0,1, \quad t>0, \\
& u(x, 0)=\psi(x), \quad 0<x<1,
\end{aligned}
$$

where $\mathcal{B}$ is a boundary operator (see below) and $\psi(x)$ and $\psi^{\prime}(x)$ are bounded with $\psi(x) \geq 0$ in $[0,1]$ (the last requirement is a consequence of the fact that for any $\psi(x)$ the solution $u$ becomes nonnegative throughout $0<x<1$ at some time $t)$. The fact that $f(u)=H(1-u)$ is a decreasing function permit us to use comparison techniques $[10,11]$, see next section.

This work follows the ideas and techniques (comparison methods) used in $[10,18]$. The presence of the convection term (as in [18]) creates more difficulties and fights against the "blow-up" of the timedependent solution. Here, in addition, we examine an asymmetric case (Dirichlet problem) which is connected with a two-parameter family of steady-states, resulting in more technical difficulties. Finally, in contrast to $[10,18,19]$, here we give a rigorous analysis of the existence and uniqueness of the time-dependent solution.

\section{Existence and uniqueness}

Since $H(1-s)$ is not strictly positive nor Lipschitz continuous the local existence of solutions to problem (3) does not follow by standard Picard type arguments [9]. Therefore we introduce a one-parameter 
family of Lipschitz continuous functions

$$
f^{\varepsilon}(s)=g\left(\frac{1-s}{\varepsilon}\right)+\varepsilon, \quad 0<\varepsilon<1,
$$

where $g(s)$ is an increasing and Lipschitz continuous function with $g(s)=1$ for $s \geq 1$ and $g(s)=0$ for $s \leq-1$. Thus

$$
f^{\varepsilon}(s) \rightarrow H(1-s) \quad \text { as } \varepsilon \rightarrow 0 \quad \text { for every } s \in \mathbb{R} .
$$

Let $u^{\varepsilon}$ be the solution of problem (3) when $f(s)$ has been replaced by $f^{\varepsilon}(s)$, then $u^{\varepsilon}$ is the unique (classical) solution in $\bar{\Omega}_{T_{\varepsilon}}=[0,1] \times$ $\left[0, T_{\varepsilon}\right]$ for some $T_{\varepsilon}>0$ (we use now the standard Picard type arguments $[11,12])$.

Also $u^{\varepsilon}$ can be written in an integral formulation:

$$
\begin{aligned}
& u^{\varepsilon}(x, t)=\int_{0}^{1} G(x-y, t) \psi(y) d y \\
& +\lambda \int_{0}^{t} \int_{0}^{1} G(x-y, t-s) \frac{f^{\varepsilon}\left(u^{\varepsilon}(y, s)\right)}{\left(\int_{0}^{1} f^{\varepsilon}\left(u^{\varepsilon}(y, s)\right) d y\right)^{2}} d y d s
\end{aligned}
$$

where $G$ is the Green function for $L:=\frac{\partial}{\partial t}-\frac{\partial^{2}}{\partial x^{2}}+\frac{\partial}{\partial x}$ and $\mathcal{B}$, in $0<t<T=\min _{0<\varepsilon<1} T_{\varepsilon}$.

Hence $u^{\varepsilon} \in C^{1}\left(\bar{\Omega}_{T}\right)$ where $\bar{\Omega}_{T}=[0,1] \times[0, T]$; moreover it is straightforwardly proved that $0<u^{\varepsilon}(x, t) \leq C$ in $\bar{\Omega}_{T}$ for some positive constant $C$. So we can use Ascoli-Arzela theorem to obtain a convergent subsequence $\left(u^{\eta}\right)_{0<\eta<1}$. By again replacing the parameter $\eta$ with $\varepsilon$, we have

$$
u^{\varepsilon}(x, t) \rightarrow u(x, t), \quad \varepsilon \rightarrow 0,
$$

uniformly in $(x, t) \in \Omega_{T}$, with $u \in C\left(\Omega_{T}\right)$. 
For clarity let

$$
h(x, t)=\frac{H(1-u(x, t))}{\left(\int_{0}^{1} H(1-u(y, t)) d y\right)^{2}} .
$$

Taking now the limit of (4) as $\varepsilon \rightarrow 0$ and applying the Lebesque dominated convergence theorem we have

$$
\begin{aligned}
u(x, t)= & \int_{0}^{1} G(x-y, t) \psi(y) d y \\
& +\lambda \int_{0}^{t} \int_{0}^{1} G(x-y, t-s) h(y, s) d y d s .
\end{aligned}
$$

Let $\Omega_{0 T}$ be the subset of $\Omega_{T}$ where the function $h(x, t)$ is discontinuous. Then $u_{t}, u_{x x}$ exist and are continuous in $\Omega_{1 T}=\Omega_{T} \backslash \Omega_{0 T}$, while $u, u_{x}$ are continuous in $\Omega_{T}$. Thus $u$ is a classical solution to problem (3) in $\Omega_{1 T}$ and a "weak" $V_{2}^{1,0}$-solution in $\Omega_{T}$. By a "weak" $V_{2}^{1,0}$-solution we mean that $u$ satisfies

$$
\begin{aligned}
& \int_{0}^{1}[u \xi]_{0}^{t} d x-\int_{0}^{t} \int_{0}^{1} u \xi_{t} d x d s \\
& -\int_{0}^{t} \int_{0}^{1}\left(u \xi_{x x}-u_{x} \xi+\lambda h(x, s) \xi\right) d x d s=0,
\end{aligned}
$$

for any $0 \leq t \leq T$ and $\xi \in C^{2}\left(\Omega_{T}\right)$ which satisfies the boundary conditions (3b). By standard parabolic theory this solution is unique provided that $\psi \in L^{\infty}([0,1])[1,13]$.

Moreover, if $v$ satisfies (6) with an inequality of $\geq(\leq)$ in place of the equality, then $v$ is called a "weak" upper (lower) solution. Now if $\bar{u}, \underline{u}$ are weak upper and lower solutions respectively to (3) in $\Omega_{T}$, then one obtains, by using maximum principle, that $\underline{u} \leq \bar{u}$ in $\Omega_{T}$. Here is where we need $f$ to be a decreasing function [11]. 
In the following we use the meaning of solution, upper (lower)solution in the sense of the above analysis, that is, instead of (6) we use (3).

\section{$3 \quad$ Asymptotic behaviour and "blow-up"}

\subsection{The mixed problem}

Consider the following problem:

$$
\begin{aligned}
& u_{t}-u_{x x}+u_{x}=\frac{\lambda H(1-u)}{\left(\int_{0}^{1} H(1-u) d x\right)^{2}}, \quad 0<x<1, t>0,(7 \mathrm{a}) \\
& u(0, t)=u_{x}(1, t)=0, \quad t>0, \\
& u(x, 0)=\psi(x), \quad 0<x<1 .
\end{aligned}
$$

Equation (7a) is equivalent to

$$
u_{t}-u_{x x}+u_{x}= \begin{cases}0, & \text { for } u \geq 1 \\ \lambda / m^{2}(t), & \text { for } u<1 .\end{cases}
$$

By $m(t)$ we denote the measure of the subset of $[0,1]$ where $u<1$, $(m(t)>0$ for $t>0$ since $u(x, t)$ is continuous with respect to $x$ and $u(0, t)=0)$.

For simplicity we consider $0 \leq \psi(x) \leq 1$ for $x \in[0,1]$, then $0 \leq u(x, t) \leq 1$ for $(x, t) \in \bar{\Omega}_{T}$, on using the maximum principle. Now we have the following:

Lemma 1 If $\psi^{\prime}(x) \geq 0$ then the solution $u(x, t)$ to problem (7) is increasing with respect to $x$. 
Proof: We consider the problem

$$
\begin{aligned}
& u_{t}^{\varepsilon}-u_{x x}^{\varepsilon}+u_{x}^{\varepsilon}=\frac{\lambda f^{\varepsilon}\left(u^{\varepsilon}\right)}{\left(\int_{0}^{1} f^{\varepsilon}\left(u^{\varepsilon}\right) d x\right)^{2}}, \quad 0<x<1,0<t<T, \text { (8a) } \\
& u^{\varepsilon}(0, t)=u_{x}^{\varepsilon}(1, t)=0, \quad 0<t<T, \\
& u^{\varepsilon}(x, 0)=\psi(x), \quad 0<x<1 .
\end{aligned}
$$

Differentiating (8a) with respect to $x$ we obtain:

$$
v_{t}^{\varepsilon}-v_{x x}^{\varepsilon}+v_{x}^{\varepsilon}-\lambda I^{\varepsilon}(t)\left(\frac{d}{d u^{\varepsilon}} f^{\varepsilon}\left(u^{\varepsilon}\right)\right) v^{\varepsilon}=0, \quad 0<x<1,0<t,
$$

where $v^{\varepsilon}=u_{x}^{\varepsilon}$ and $I^{\varepsilon}(t)=\left(\int_{0}^{1} f^{\varepsilon}\left(u^{\varepsilon}\right) d x\right)^{-2}$. Thus by using the maximum principle we have $v^{\varepsilon}(x, t)=u_{x}^{\varepsilon}(x, t) \geq 0$ for $(x, t) \in \Omega_{T}$ and $0<\varepsilon<1$. Hence

$$
u^{\varepsilon_{k}}(x, t) \leq u^{\varepsilon_{k}}(y, t), \quad x<y, 0<t<T,
$$

where $u^{\varepsilon_{\kappa}} \rightarrow u, \varepsilon_{\kappa} \rightarrow 0$ as $\kappa \rightarrow \infty, \kappa \in \mathbb{N}$. This implies that $u$ is an increasing function of $x$.

Now we distinguish two cases:

1. $u<1$ for every $x$ in $[0,1]$, then equation (7a) becomes

$$
u_{t}=u_{x x}-u_{x}+\lambda, \quad 0<x<1, t>0 .
$$

The corresponding steady problem is

$$
\begin{aligned}
& w^{\prime \prime}-w^{\prime}+\lambda=0, \quad 0<x<1, \\
& w(0)=w^{\prime}(1)=0 .
\end{aligned}
$$


2. $u=1$ in a subset of $[0,1]$, then Lemma 1 implies

$$
\begin{aligned}
& u_{t}=u_{x x}-u_{x}+\frac{\lambda}{r^{2}(t)}, \quad 0<x<r(t), t>0 \\
& u=1, \quad u_{x}=0, \quad r(t) \leq x \leq 1, t>0 \\
& u(0, t)=u_{x}(1, t)=0, \quad t>0 \\
& u(x, 0)=\psi(x), \quad 0<x<1
\end{aligned}
$$

where $0<r=r(t)<1$. The fact that $u_{x}(r, t)=0$ follows from the continuity of $u_{x}$ at $x=r$ (see the previous section). In this case there exists a one-parameter family of steady states of the form:

$$
\begin{aligned}
& w^{\prime \prime}-w^{\prime}+\frac{\lambda}{s^{2}}=0, \quad 0<x<s, \\
& w(x)=1, \quad w^{\prime}(x)=0, \quad s \leq x \leq 1, \\
& w(0)=w^{\prime}(1)=0,
\end{aligned}
$$

where $0<s<1$.

The solution to problem (10) is

$$
w_{1}(x ; \lambda)=\frac{\lambda}{e}\left(1-e^{x}+x e\right)
$$

and $\max _{[0,1]} w_{1}(x)=w_{1}(1)=\frac{\lambda}{e}<1$, so $\lambda<\lambda^{*}=e$. On the other hand, the one-parameter family of problems (12) has the solution:

$$
\begin{aligned}
& w_{2}(x ; s)=\frac{\lambda(s)}{s^{2} e^{s}}\left(1-e^{x}+x e^{s}\right), \quad 0 \leq x<s, \\
& w_{2}(x ; s)=1, \quad w_{2}^{\prime}(x ; s)=0, \quad s \leq x \leq 1 .
\end{aligned}
$$

Since $w_{2}(s ; s)=1$ we obtain

$$
\lambda(s)=\frac{s^{2} e^{s}}{1-e^{s}+s e^{s}} .
$$


The latter implies that

$$
\begin{aligned}
& w_{2}(x ; s)=\frac{1-e^{x}+x e^{s}}{1-e^{s}+s e^{s}}, \quad 0 \leq x<s \\
& w_{2}(x ; s)=1, \quad w_{2}^{\prime}(x ; s)=0, \quad s \leq x \leq 1 .
\end{aligned}
$$

The function $\lambda(s)$ is increasing. Indeed

$$
\lambda^{\prime}(s)=\frac{s e^{s} h(s)}{\left(1-e^{s}+s e^{s}\right)^{2}}>0,
$$

where $h(s)=2-2 e^{s}+s e^{s}+s$ and $h(s)>h(0)=0$, for $0<s<1$. Moreover,

$$
\begin{aligned}
& \lambda(1-)=\lim _{s \rightarrow 1-} \lambda(s)=\lim _{s \rightarrow 1-} \frac{s^{2} e^{s}}{1-e^{s}+s e^{s}}=e=\lambda^{*}, \\
& \lambda(0+)=\lim _{s \rightarrow 0+} \lambda(s)=\lim _{s \rightarrow 0+} \frac{s^{2} e^{s}}{1-e^{s}+s e^{s}}=\lim _{s \rightarrow 0+}(2+s)=2=\lambda_{*} .
\end{aligned}
$$

We also observe that

$$
\begin{aligned}
& w_{1}^{\prime}(0 ; \lambda)=\frac{\lambda}{e}(e-1), \quad 0<\lambda<\lambda^{*}=e, \\
& w_{2}^{\prime}(0 ; 1-)=\lim _{s \rightarrow 1-} w_{2}^{\prime}(0 ; s)=e-1, \quad \lim _{s \rightarrow 0+} w_{2}^{\prime}(0 ; s)=\infty,
\end{aligned}
$$

since $\lambda(s) \rightarrow e$ as $s \rightarrow 1-$. Finally we obtain the response diagram of Figure 1.

\subsubsection{Stability}

We now study the stability of steady-states to problem (7) for $0<$ $\lambda \leq \lambda^{*}=e$ by using comparison methods.

In the case where $0<\psi(x) \leq w_{1}(x)$ the function

$$
z(x, t)=\frac{b(t)}{e}\left(1-e^{x}+x e\right), \quad 0 \leq x \leq 1, t>0,
$$




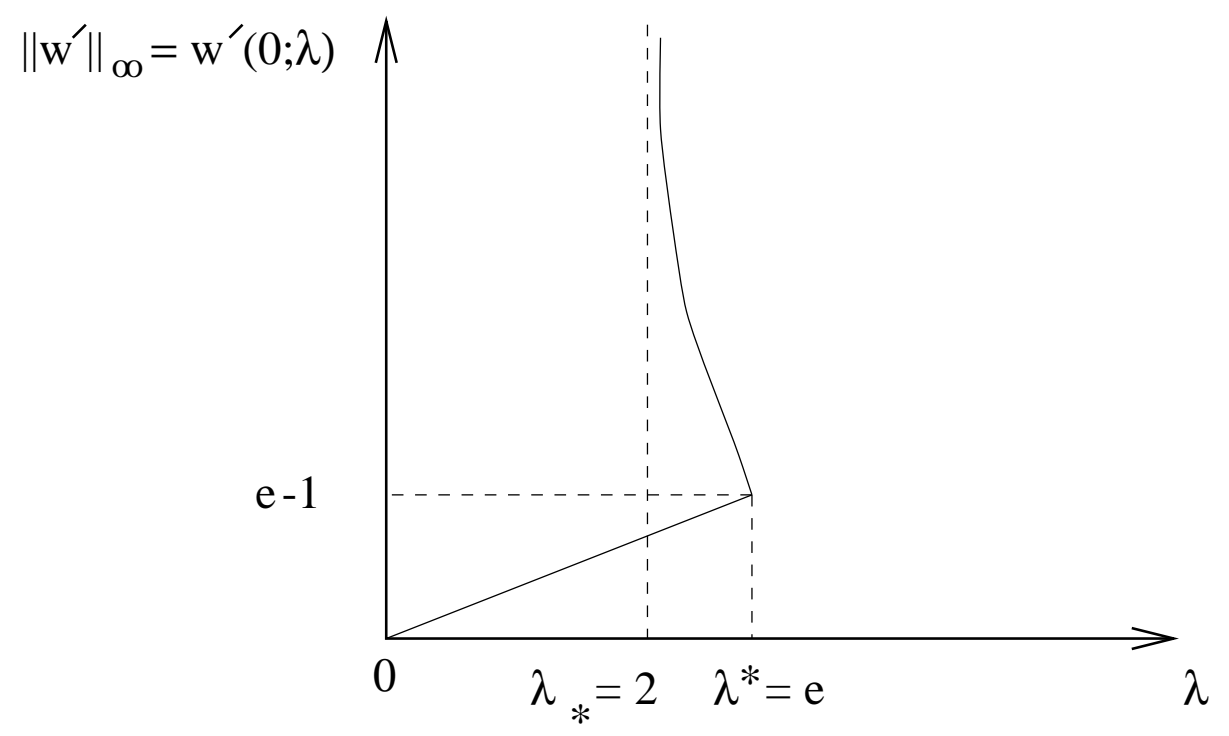

Figure 1: The non-local response diagram to problems (11) and (12). 
is a lower solution to (7) for $0<\lambda \leq \lambda_{*}$ provided that $b(t)$ satisfies

$$
\frac{d b}{d t}=\dot{b}(t)=\lambda-b(t)>0, \quad t>0, \quad 0 \leq b(0)=b_{0} \leq \lambda,
$$

or

$$
b(t)=\lambda+\left(b_{0}-\lambda\right) e^{-t} .
$$

Then $z(x, t) \leq u(x, t) \leq w_{1}(x)$ for $0 \leq x \leq 1$ and $b(t) \rightarrow \lambda$ - as $t \rightarrow \infty$, whence $z(x, t) \rightarrow w_{1}(x)$ as $t \rightarrow \infty$ uniformly in $x$. The same result holds for $u$, that is, $u(x, t) \rightarrow w_{1}(x)-$ as $t \rightarrow \infty$ uniformly in $x$.

If now $w_{1}(x) \leq \psi(x) \leq 1$ then the function

$$
U(x, t)=w_{2}(x ; r)= \begin{cases}\frac{1-e^{x}+x e^{r}}{1-e^{r}+r e^{r}}, & 0 \leq x<r=r(t), \\ 1, & r \leq x \leq 1,\end{cases}
$$

for $0<t<t_{1}$ and

$$
U(x, t)=w_{1}(x ; \alpha(t))=\frac{\alpha(t)}{e}\left(1-e^{x}+x e\right), \quad 0 \leq x \leq 1, t \geq t_{1},
$$

where $r\left(t_{1}\right)=1$ and $\alpha\left(t_{1}\right)=e$, is an upper solution to problem (7). The former holds as long as

$$
U_{r}(x ; r)=\frac{e^{r}\left(x-r+r e^{x}-x e^{r}\right)}{\left(1-e^{r}+r e^{r}\right)^{2}}<0 \quad \text { for } 0<x<r=r(t),
$$

and

$$
\dot{r}(t)=h(r) \equiv \frac{[\lambda(r)-\lambda]\left(1-e^{r}+r e^{r}\right)^{2}}{r^{4} e^{2 r}}, \quad 0<t<t_{1}, r(0)=r_{0},
$$

where $r_{0}>0$ so that $\lambda\left(r_{0}\right)>\lambda$ and $U(x, 0)=w_{2}\left(x ; r_{0}\right) \geq \psi(x)$. Problem (20) has a unique solution, since the same holds for its equivalent transcendental equation for $r(t)$ :

$$
\int_{r_{0}}^{r(t)} \frac{\sigma^{4} e^{2 \sigma} d \sigma}{[\lambda(\sigma)-\lambda]\left(1-e^{\sigma}+\sigma e^{\sigma}\right)^{2}}=t, \quad 0<t<t_{1} .
$$


Note that the function

$$
G(\xi)=\int_{r_{0}}^{\xi} \frac{\sigma^{4} e^{2 \sigma} d \sigma}{[\lambda(\sigma)-\lambda]\left(1-e^{\sigma}+\sigma e^{\sigma}\right)^{2}}
$$

is a $C^{1}$-diffeomorphism from $\left[r_{0}, 1\right]$ to $[0, T]$ with

$$
T=\int_{r_{0}}^{1} \frac{\sigma^{4} e^{2 \sigma} d \sigma}{[\lambda(\sigma)-\lambda]\left(1-e^{\sigma}+\sigma e^{\sigma}\right)^{2}}<\infty,
$$

see [6]. We also require that

$$
\dot{\alpha}(t)=\lambda-\alpha(t), \quad t>t_{1}, \quad \alpha\left(t_{1}\right)=e,
$$

or equivalently

$$
\alpha(t)=\lambda+(e-\lambda) e^{t_{1}-t} \rightarrow \lambda+\quad \text { as } t \rightarrow \infty .
$$

Thus $w_{1}(x) \leq u(x, t) \leq U(x, t)$ for $(x, t) \in[0,1] \times[0, \infty)$ and $u(x, t) \rightarrow w_{1}(x)$ as $t \rightarrow \infty$ uniformly in $x$. Finally we obtain that for $0<\lambda \leq \lambda_{*}=2$ the unique steady state $w_{1}(x)$ is asymptotically stable for all $0<\psi(x) \leq 1$.

For the case $2=\lambda_{*}<\lambda<\lambda^{*}=e$, we have two steady solutions $w_{1}(x), w_{2}(x):=w_{2}(x ; s)$ (for $\lambda=\lambda^{*}, w_{1}(x)=w_{2}(x)$ ). Following the same steps as above, we prove that $w_{1}(x)$ is asymptotically stable and attracts solutions with initial condition $0<\psi(x)<w_{2}(x)$.

Now we take $w_{2}(x)<\psi(x) \leq 1$ and prove that, for $t>0$,

$$
z(x, t)= \begin{cases}\frac{1-e^{x}+x e^{r}}{1-e^{r}+r e^{r}}, & 0 \leq x<r=r(t), \\ 1, & r \leq x \leq 1,\end{cases}
$$

is a lower solution to (7) as long as $r(t)$ satisfies

$$
\dot{r}(t)=h(r) \equiv \frac{[\lambda(r)-\lambda]\left(1-e^{r}+r e^{r}\right)^{2}}{r^{4} e^{2 r}}, \quad t>0, \quad r(0)=r_{0},
$$


where $0<r_{0}<s$, so that $z(x, 0)=w_{2}\left(x ; r_{0}\right)<\psi(x)$.

The previous problem is equivalent to the transcendental equation for $r(t)$

$$
\int_{r(t)}^{r_{0}} \frac{\sigma^{4} e^{2 \sigma} d \sigma}{[\lambda-\lambda(\sigma)]\left(1-e^{\sigma}+\sigma e^{\sigma}\right)^{2}}=\int_{r(t)}^{r_{0}} \frac{d \sigma}{g(\sigma)}=t
$$

where $g(\sigma)=-h(\sigma)$ and $t$ takes its maximum $T^{*}$ when $r(t)$ becomes zero. Here

$$
T^{*}=\int_{0}^{r_{0}} \frac{d \sigma}{g(\sigma)}<\infty
$$

(the latter holds since $g(0)=4 /(\lambda-2), \lambda>2$, is bounded). This implies that $u(x, t) \rightarrow 1-$ as $t \rightarrow t^{*}-\leq T^{*}$ for every $x \in(0,1]$ and $u_{x}(0, t) \rightarrow \infty$ as $t \rightarrow t^{*}-$, since $z(x, t) \leq u(x, t)$. Hence for $\lambda_{*}<\lambda \leq \lambda^{*}$ the maximum steady state $w_{2}(x)$ is unstable; more precisely the solution $u(x, t)$ to $(7)$ "blows up" in the sense that $u$ becomes 1 in $(0,1]$ at a finite time (this could be an indication that the mathematical model fails to describe the physical problem).

\subsection{2 "Blow-up" for $\lambda>\lambda^{*}=e$}

We now prove that the solution $u(x, t)$ "blows up" for $\lambda>\lambda^{*}=$ $e$. Therefore we consider the function

$$
z(x, t)=\frac{\alpha(t)}{e}\left(1-e^{x}+x e\right), \quad 0 \leq x \leq 1,0<t<t_{1},
$$

where $0 \leq \alpha(t) \leq e$ satisfies

$$
\dot{\alpha}(t)=\lambda-\alpha(t)>0, \quad t>0, \quad \alpha(0)=0,
$$

and $\alpha\left(t_{1}\right)=e\left(t_{1}=\ln \lambda-\ln (\lambda-e)<\infty\right)$. Assuming that $u$ "exists" $(u<1$ somewhere in $[0,1])$ for $t=t_{1}$ we consider $z$ for 
$t \geq t_{1}$

$$
z(x, t)=\left\{\begin{array}{ll}
\frac{1-e^{x}+x e^{r}}{1-e^{r}+r e^{r}}, & 0 \leq x<r=r(t), \\
1, & r \leq x \leq 1,
\end{array} \quad t>t_{1},\right.
$$

where $r\left(t_{1}\right)=1$.

The function $z$ is a lower solution to (7) provided that $r(t)$ satisfies:

$$
\dot{r}(t)=h(r) \equiv \frac{[\lambda(r)-\lambda]\left(1-e^{r}+r e^{r}\right)^{2}}{r^{4} e^{2 r}}, \quad t>t_{1}, \quad r\left(t_{1}\right)=1 .
$$

Problem (25) is equivalent to the transcendental equation for $r(t)$

$$
t_{1}+\int_{r(t)}^{1} \frac{d \sigma}{g(\sigma)}=t_{1}+\int_{r(t)}^{1} \frac{\sigma^{4} e^{2 \sigma} d \sigma}{[\lambda-\lambda(\sigma)]\left(1-e^{\sigma}+\sigma e^{\sigma}\right)^{2}}=t .
$$

Again there exists a

$$
T_{1}^{*}=\int_{0}^{1} \frac{d \sigma}{g(\sigma)}+t_{1}<\infty,
$$

such that $r\left(T_{1}^{*}\right)=0$. Thus $u(x, t) \rightarrow 1$ as $t \rightarrow t_{1}^{*}-\leq T_{1}^{*}<\infty$ for every $x \in(0,1]$ and $u_{x}(0, t) \rightarrow \infty$ as $t \rightarrow t_{1}^{*}-$, that is, $u$ "blows up" in finite time $t_{1}^{*}$.

\subsection{The Dirichlet problem}

Now we consider the problem

$$
\begin{aligned}
& u_{t}-u_{x x}+u_{x}=\frac{\lambda H(1-u)}{\left(\int_{0}^{1} H(1-u) d x\right)^{2}}, \quad 0<x<1, t>0,(26 \mathrm{a}) \\
& u(0, t)=u(1, t)=0, \quad t>0, \\
& u(x, 0)=\psi(x), \quad 0<x<1 .
\end{aligned}
$$


This problem is asymmetric due to the convection term. More precisely equation $(26 \mathrm{a})$ is written:

$$
u_{t}-u_{x x}+u_{x}= \begin{cases}0, & \text { for } u \geq 1, \\ \lambda / n^{2}(t), & \text { for } u<1,\end{cases}
$$

denoting with $n(t)$ the measure of the subset of $[0,1]$ where $u<1$.

On taking initial data $0 \leq \psi(x) \leq 1$ we obtain by the maximum principle that $0 \leq u(x, t) \leq 1$.

Again we distinguish two cases:

1. $u<1$ for every $x$ in $[0,1]$, then equation (26a) becomes

$$
u_{t}=u_{x x}-u_{x}+\lambda, \quad 0<x<1, t>0,
$$

while the corresponding steady problem is

$$
\begin{aligned}
& w^{\prime \prime}-w^{\prime}+\lambda=0, \quad 0<x<1, \\
& w(0)=w(1)=0 .
\end{aligned}
$$

2. $u=1$ in a subset of $[0,1]$, then there exist $0<s_{1} \leq s_{2}<1$ such that the corresponding steady problem has the form

$$
\begin{aligned}
& w^{\prime \prime}-w^{\prime}+\frac{\lambda}{\left(1+s_{1}-s_{2}\right)^{2}}=0, \\
& \quad \text { for } \quad 0<x<s_{1} \quad \text { or } \quad s_{2}<x<1, \\
& w(x)=1, \quad s_{1} \leq x \leq s_{2}, \\
& w(0)=w(1)=0 .
\end{aligned}
$$

Our purpose now is to find the response diagram to problems (28) and (29). The solution to (28) is $w_{1}(x)=\frac{\lambda}{e-1}\left(1-e^{x}+x e-x\right)$. It 
is easily seen that at $x_{0}=\ln (e-1)$ the solution $w_{1}$ takes its (unique) maximum

$$
w_{1}\left(x_{0}\right)=\lambda\left[\frac{2-e+(e-1) \ln (e-1)}{e-1}\right] .
$$

Since $w_{1}\left(x_{0}\right)<1$, we deduce that

$$
\lambda<\frac{e-1}{2-e+(e-1) \ln (e-1)}=\lambda^{*} \simeq 8.11019739 .
$$

On the other hand, the two-parameter solution to (29) is

$$
w_{2}\left(x ; s_{1}, s_{2}\right)= \begin{cases}\frac{\lambda\left(1-e^{x}+x e^{s_{1}}\right)}{\left(1+s_{1}-s_{2}\right)^{2} e^{s_{1}}}, & 0 \leq x<s_{1}, \\ 1, & s_{1} \leq x \leq s_{2}, \\ \frac{\lambda\left(e-e^{s_{2}}-e^{x}+x e^{s_{2}}\right)}{\left(1+s_{1}-s_{2}\right)^{2} e^{s_{2}}}, & s_{2}<x \leq 1 .\end{cases}
$$

However $w_{2}\left(s_{1} ; s_{1}, s_{2}\right)=1$, so the first branch implies

$$
\lambda\left(s_{1}, s_{2}\right)=\frac{\left(1+s_{1}-s_{2}\right)^{2} e^{s_{1}}}{1+\left(s_{1}-1\right) e^{s_{1}}},
$$

while the third branch gives

$$
\lambda\left(s_{1}, s_{2}\right)=\frac{\left(1+s_{1}-s_{2}\right)^{2} e^{s_{2}}}{e+\left(s_{2}-2\right) e^{s_{2}}} .
$$

The relations (30) and (31) imply

$$
e^{-s_{1}}+s_{1}-1=e^{1-s_{2}}+s_{2}-2,
$$

provided that $s_{1} \neq 0, s_{2} \neq 1$.

It is easily proved that $s_{1} \rightarrow 0+$ as $s_{2} \rightarrow 1-, s_{1} \rightarrow x_{0}-$ as $s_{2} \rightarrow x_{0}+$ and vice versa. 
From (32) we obtain $F\left(s_{1}, s_{2}\right)=e^{1-s_{2}}+s_{2}-1-e^{-s_{1}}-s_{1}=0$ for $\left(s_{1}, s_{2}\right) \in\left(0, x_{0}\right) \times\left(x_{0}, 1\right)$. Also $\partial F\left(s_{1}, s_{2}\right) / \partial s_{2}=1-e^{1-s_{2}} \neq 0$, then from the implicit function theorem we get $s_{2}=\phi\left(s_{1}\right)$ for all $\left(s_{1}, s_{2}\right) \in\left(0, x_{0}\right) \times\left(x_{0}, 1\right)$ and

$$
\phi^{\prime}\left(s_{1}\right)=\frac{1-e^{-s_{1}}}{1-e^{1-s_{2}}}<0 .
$$

Now we examine the monotonicity of $\lambda$. By using (33) we have

$$
\begin{aligned}
\frac{\partial}{\partial s_{1}} \lambda\left(s_{1}, s_{2}\right)= & \frac{\left(1+s_{1}-\phi\left(s_{1}\right)\right)^{2}}{\left(e^{-s_{1}}+s_{1}-1\right)^{2}\left(e^{1-s_{2}}-1\right)}\left[2\left(e^{-s_{1}}+s_{1}-1\right)\right. \\
& \left.-\left(1-e^{-s_{1}}\right)\left(e^{1-s_{2}}-1\right)\right] \\
= & K\left(s_{1}, s_{2}\right) \Lambda\left(s_{1}, s_{2}\right)
\end{aligned}
$$

where

$$
K\left(s_{1}, s_{2}\right)=\frac{\left(1+s_{1}-\phi\left(s_{1}\right)\right)^{2}}{\left(e^{-s_{1}}+s_{1}-1\right)^{2}\left(e^{1-s_{2}}-1\right)}>0,
$$

and

$$
\begin{aligned}
\Lambda\left(s_{1}, s_{2}\right) & =2\left(e^{-s_{1}}+s_{1}-1\right)-\left(1-e^{-s_{1}}\right)\left(e^{1-s_{2}}-1\right) \\
& =e^{-s_{1}}+s_{1}-2+s_{2}+e^{-2 s_{1}}+s_{1} e^{-s_{1}}-s_{2} e^{-s_{1}} .
\end{aligned}
$$

The function $\Lambda\left(s_{1}, s_{2}\right)$ takes its minimum at the two points $\left(0, x_{0}\right)$ and $(0,1)$. But only the point $(0,1)$ satisfies the restriction (32). So $\Lambda\left(s_{1}, s_{2}\right)>\Lambda(0,1)=0$ in $\left(0, x_{0}\right) \times\left(x_{0}, 1\right)$. Finally we have

$$
\frac{\partial}{\partial s_{2}} \lambda\left(s_{1}, s_{2}\right)>0 \quad \text { and } \quad \frac{\partial}{\partial s_{2}} \lambda\left(s_{1}, s_{2}\right)=\frac{\partial}{\partial s_{2}} \lambda\left(s_{1}, s_{2}\right) \frac{1}{\phi^{\prime}\left(s_{1}\right)}<0
$$

for $\left(s_{1}, s_{2}\right) \in\left(0, x_{0}\right) \times\left(x_{0}, 1\right)$. We also have

$$
\lim _{s_{1} \rightarrow x_{0}} \lambda\left(s_{1}, s_{2}\right)=\frac{e-1}{1+(e-1)[\ln (e-1)-1]}=\lambda^{*}
$$


and

$$
\lim _{s_{1} \rightarrow 0} \lambda\left(s_{1}, s_{2}\right)=\lim _{s_{1} \rightarrow 0} \frac{2\left(1+s_{1}-\phi\left(s_{1}\right)\right)\left(1-\phi^{\prime}\left(s_{1}\right)\right)}{1-e^{-s_{1}}} .
$$

After some calculations we finally obtain

$$
\lim _{s_{1} \rightarrow 0} \lambda\left(s_{1}, s_{2}\right)=4 \lim _{s_{1} \rightarrow 0} \frac{1-\phi^{\prime}\left(s_{1}\right)}{e^{-s_{1}}}=8=\lambda_{*}
$$

(we have used that $\lim _{s \rightarrow 0} \phi^{\prime}(s)=-1$ ).

In addition we take

$$
\begin{aligned}
& w_{1}^{\prime}(0 ; \lambda)=\frac{\lambda(e-2)}{e-1} \quad \text { for } 0<\lambda \leq \lambda^{*}, \\
& w_{2}^{\prime}\left(0 ; x_{0}, x_{0}\right)=w_{2}^{\prime}\left(0 ; \lambda^{*}\right)=\frac{\lambda^{*}(e-2)}{e-1}, \\
& w_{2}^{\prime}(0 ; 0,1)=w_{2}^{\prime}\left(0 ; \lambda_{*}\right)=\lim _{s_{1} \rightarrow 0} \frac{e^{s_{1}}-1}{1+s_{1} e^{s_{1}}-e^{s_{1}}}=\infty .
\end{aligned}
$$

From the above analysis we obtain the response diagram of Figure 2.

\subsubsection{Stability}

We now study the stability of steady-states for $0<\lambda \leq \lambda^{*}=$ 8.11019739. Firstly we consider $0<\lambda \leq \lambda_{*}=8$, then $w_{1}(x)$ is the unique steady solution.

Again we prove, for $0 \leq \psi(x)<w_{1}(x)$, that

$$
z(x, t)=\frac{b(t)}{e-1}\left(1-e^{x}+x e-x\right), \quad 0 \leq x \leq 1, t>0,
$$

is a lower solution to (26) provided that $0 \leq b(t) \leq \lambda$ and $b(t)$ satisfies

$$
\frac{d b}{d t}=\dot{b}(t)=\lambda-b(t)>0, \quad t>0, \quad b(0)=b_{0} \geq 0,
$$




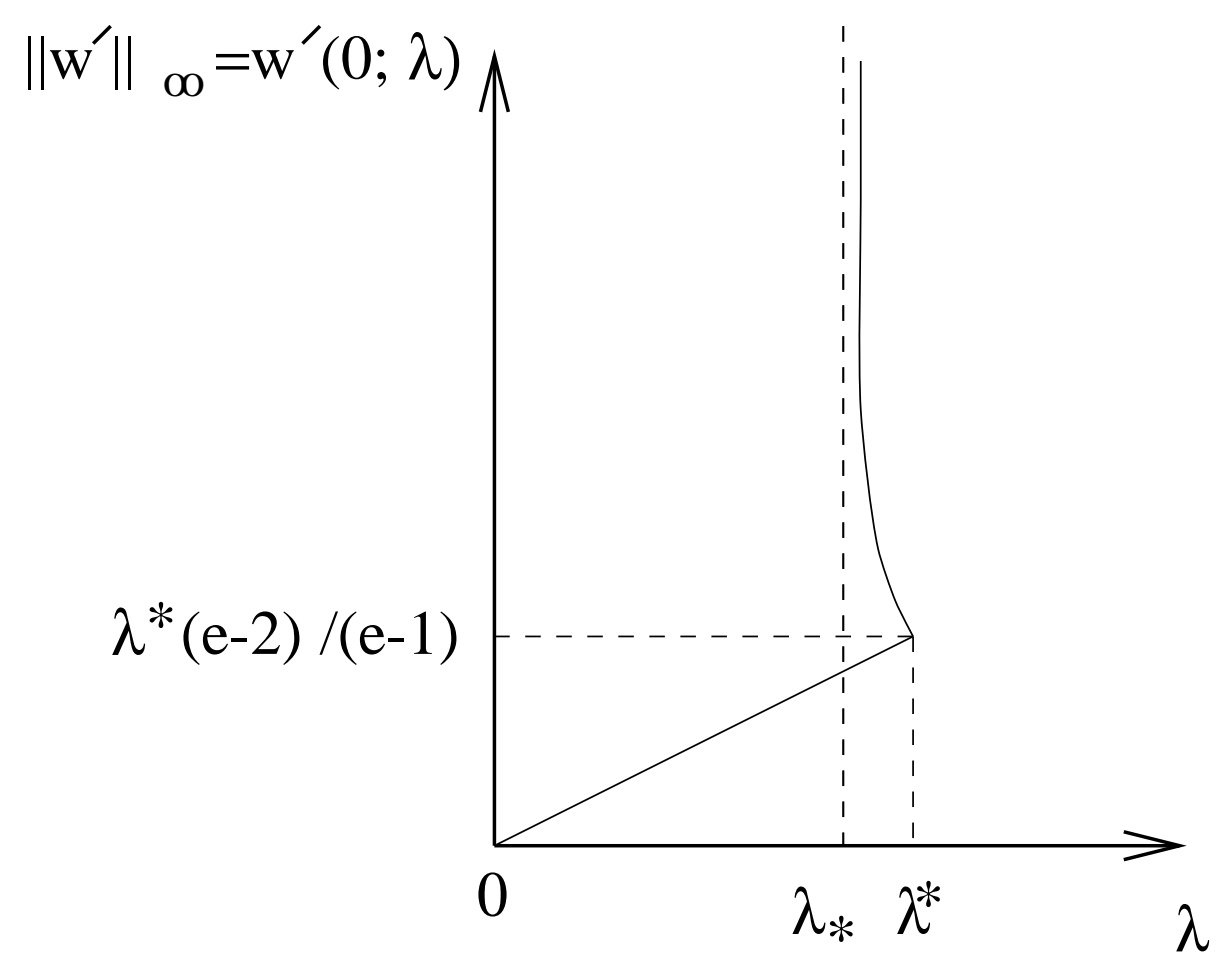

Figure 2: The non-local response diagram to problems (28) and $(29) ; \lambda_{*}=8, \lambda^{*}=8.11019739$. 
or equivalently $b(t)=\lambda+\left(b_{0}-\lambda\right) e^{-t} \rightarrow \lambda-$. Hence $u(x, t) \rightarrow w_{1}(x)$ as $t \rightarrow \infty$ uniformly in $x$.

For $w_{1}(x)<\psi(x) \leq 1$, our prospective comparison function $U(x, t)$ is

$$
U(x, t)=w_{2}\left(x ; r_{1}, r_{2}\right)= \begin{cases}\frac{1+x e^{r_{1}}-e^{x}}{1+r_{1} e^{r_{1}}-e^{r_{1}}}, & 0 \leq x<r_{1}, \\ 1, & r_{1} \leq x \leq r_{2}, \\ \frac{e+x e^{r_{2}}-e^{r_{2}}-e^{x}}{e+\left(r_{2}-2\right) e^{r_{2}}}, & r_{2}<x \leq 1,\end{cases}
$$

where $r_{1}$ and $r_{2}$ are functions of $t, 0<t<t_{1}$, and

$$
U(x, t)=w_{1}(x ; \alpha(t))=\frac{\alpha(t)}{e-1}\left(1-e^{x}+x e-x\right), \quad 0 \leq x \leq 1,
$$

for $t \geq t_{1}$. Also the functions $r_{1}$ and $r_{2}$ with $0<r_{1}(t)<r_{2}(t)<1$ satisfy relation (32). Both relation (32) and problem

$$
\dot{r}_{1}(t)=h\left(r_{1}\right) \equiv \frac{\left[\lambda\left(r_{1}, \phi\left(r_{1}\right)\right)-\lambda\right]\left(1-e^{r_{1}}+r_{1} e^{r_{1}}\right)^{2}}{r_{1}^{2} e^{2 r_{1}}\left(1+r_{1}-\phi\left(r_{1}\right)\right)^{2}}, \quad 0<t<t_{1},
$$

$$
r_{1}(0)=\hat{r}_{0}
$$

$\left(\hat{r}_{0}: \lambda\left(\hat{r}_{0}, \phi\left(\hat{r}_{0}\right)\right)>\lambda\right.$ and $\left.U(x, 0)=w_{2}\left(x ; \hat{r}_{0}, \phi\left(\hat{r}_{0}\right)\right) \geq \psi(x)\right)$ imply that $U(x, t)$ is an upper solution to problem (26).

The existence and uniqueness of (37) is derived using similar arguments to the mixed problem.

For $t>t_{1}$ we require $\alpha(t)$ to satisfy

$$
\dot{\alpha}(t)=\lambda-\alpha(t)<0, \quad t>t_{1}, \quad \alpha\left(t_{1}\right)=\lambda^{*},
$$


or equivalently $\alpha(t)=\lambda+\left(\lambda^{*}-\lambda\right) e^{t_{1}-t} \rightarrow \lambda+$ as $t \rightarrow \infty$. Thus, it is proved that $u(x, t) \rightarrow w_{1}(x)+$ as $t \rightarrow \infty$ uniformly in $x$. So $u(x, t)$ is a global in time solution for $0<\lambda \leq \lambda_{*}$ and $w_{1}(x ; \lambda)$ is asymptotically stable for all $0<\psi(x)<1$.

In the case where $\lambda_{*}<\lambda<\lambda^{*}$, if we consider initial data $0 \leq \psi(x)<w_{1}(x)$, then using similar arguments as above we prove that $u(x, t) \rightarrow w_{1}(x)-$ as $t \rightarrow \infty$ uniformly in $x$. While for initial data $w_{1}(x)<\psi(x)<w_{2}(x):=w_{2}\left(x ; s_{1}, s_{2}\right)$ we construct an upper solution similar to this defined by (35), (36). Hence, in this case, $w_{1}(x)$ is asymptotically stable.

If now $w_{2}(x)<\psi(x)$, then we construct a lower solution $z(x, t)$ of the form:

$$
z(x, t)=w_{2}\left(x ; r_{1}, r_{2}\right)= \begin{cases}\frac{1+x e^{r_{1}}-e^{x}}{1+r_{1} e^{r_{1}}-e^{r_{1}}}, & 0 \leq x<r_{1}, \\ 1, & r_{1} \leq x \leq r_{2}, \\ \frac{e+x e^{r_{2}}-e^{r_{2}}-e^{x}}{e+\left(r_{2}-2\right) e^{r_{2}}}, & r_{2}<x \leq 1,\end{cases}
$$

where $0<r_{1}(t) \leq r_{2}(t)<1$ satisfy restriction (32). Moreover $r_{1}(t)$ is the unique solution to the problem

$$
\dot{r}_{1}(t)=h\left(r_{1}\right) \equiv \frac{\left[\lambda\left(r_{1}, \phi\left(r_{1}\right)\right)-\lambda\right]\left(1-e^{r_{1}}+r_{1} e^{r_{1}}\right)^{2}}{r_{1}^{2} e^{2 r_{1}}\left(1+r_{1}-\phi\left(r_{1}\right)\right)^{2}}, \quad t>0,
$$

$$
r_{1}(0)=\hat{r}_{0},
$$

$\left(0<\hat{r}_{0}<s_{1}: z(x, 0)=w_{2}\left(x ; \hat{r}_{0}, \phi\left(\hat{r}_{0}\right)\right)<\psi(x)\right)$. Using the transcendental equation for $r_{1}(t)$

$$
\int_{r_{1}(t)}^{\hat{r}_{0}} \frac{d \sigma}{g(\sigma)}=t, \quad t>0
$$


$(g(\sigma)=-h(\sigma)>0)$ which is equivalent to problem (39) we prove the existence of $T^{*}=\int_{0}^{\hat{r}_{0}} \frac{d \sigma}{g(\sigma)}<\infty$, such that $r_{1}\left(T^{*}\right)=0$ (or equivalently $\left.r_{2}\left(T^{*}\right)=1\right)$. Whence $u(x, t) \rightarrow 1$ as $t \rightarrow t^{*}-\leq T^{*}<$ $\infty$ for every $x \in(0,1)$ and $\left|u_{x}(\xi, t)\right| \rightarrow \infty$ as $t \rightarrow t^{*}-$ for $\xi=0,1$, that is $u(x, t)$ "blows up" in finite time. This implies that the greater steady-state $w_{2}(x)$ is unstable.

\subsection{2 "Blow-up" for $\lambda>\lambda^{*}$}

We now examine the case where $\lambda>\lambda^{*}$. We prove that $u(x, t)$ "blows up" in finite time. Therefore we consider the comparison function

$$
z(x, t)=\frac{\alpha(t)}{e-1}\left(1-e^{x}+x e-x\right), \quad 0 \leq x \leq 1,0<t<t_{1},
$$

with $0 \leq \alpha(t) \leq \lambda^{*}$. The function $z(x, t)$ is a lower solution to problem (26) provided $\alpha(t)$ satisfies:

$$
\dot{\alpha}(t)=\lambda-\alpha(t)>0, \quad 0<t<t_{1}, \quad \alpha(0)=0,
$$

where $t_{1}$ is such that $\alpha\left(t_{1}\right)=\lambda^{*}\left(t_{1}=\ln \lambda-\ln \left(\lambda-\lambda^{*}\right)<\infty\right.$, since $\left.\lambda>\lambda^{*}\right)$. If, on the other hand, $u(x, t)$ "exists" $(u<1)$ at $t=t_{1}$ then we can define $z$ for $t>t_{1}$ so that

$$
z(x, t)= \begin{cases}\frac{1-e^{x}+x e^{r_{1}}}{1-e^{r_{1}}+r_{1} e^{r_{1}}}, & 0 \leq x<r_{1}, \\ 1, & r_{1} \leq x \leq r_{2}, \\ \frac{e+x e^{r_{2}}-e^{r_{2}}-e^{x}}{e+\left(r_{2}-2\right) e^{r_{2}}}, & r_{2}<x \leq 1,\end{cases}
$$

where $0<r_{1}(t) \leq r_{2}(t)<1$ satisfy $(32)$. 
The function $z$ is a lower solution to problem (26) provided that $r_{1}(t)$ with $0<r_{1}(t)<1$, satisfies the problem

$$
\begin{gathered}
\dot{r}_{1}(t)=h\left(r_{1}\right)=\frac{\left[\lambda\left(r_{1}, \phi\left(r_{1}\right)\right)-\lambda\right]\left(1-e^{r_{1}}+r_{1} e^{r_{1}}\right)^{2}}{r_{1}^{2} e^{2 r_{1}}\left(1+r_{1}-\phi\left(r_{1}\right)\right)^{2}}, \quad t \geq t_{1}, \\
r_{1}\left(t_{1}\right)=x_{0} .
\end{gathered}
$$

Again we prove that there exists

$$
T_{1}^{*}=\int_{0}^{x_{0}} \frac{d \sigma}{g(\sigma)}+t_{1}<\infty
$$

where

$$
g(\sigma)=\frac{\sigma^{2} e^{2 \sigma}(1+\sigma-\phi(\sigma))^{2}}{[\lambda-\lambda(\sigma, \phi(\sigma))]\left(1-e^{\sigma}+\sigma e^{\sigma}\right)^{2}}
$$

such that $r_{1}\left(T_{1}^{*}\right)=0$ (or equivalently $r_{2}\left(T_{1}^{*}\right)=1$ ).

The latter implies that $u(x, t) \rightarrow 1$ as $t \rightarrow t_{1}^{*}-\leq T_{1}^{*}$ uniformly in $x \in(0,1)$ and $\left|u_{x}(\xi, t)\right| \rightarrow \infty$ as $t \rightarrow t_{1}^{*}-$, where $\xi=0,1$.

In the last part of this work we consider the case where $f(s)=$ $H(s-1)$. Now the problem is non-trivial only if $u \geq 1$ in a subset of $[0,1]$. In order to ensure this we assume that the boundary conditions are of Neumann type and $\psi(x) \geq 1$; then by using the maximum principle we obtain that $u \geq 1$ for $x \in[0,1]$ and $t>0$. Thus the problem becomes

$$
\begin{aligned}
& u_{t}-u_{x x}+u_{x}=\lambda, \quad 0<x<1, t>0, \\
& u_{x}(0,1)=u_{x}(1, t)=0, \quad t>0 \\
& u(x, 0)=\psi(x), \quad 0<x<1
\end{aligned}
$$

It is known from the classical parabolic theory that problem (43) has a unique solution. Denoting by $M(t)=\max _{[0,1]} u(\cdot, t)$, from (43a) 
we get $d M / d t=\dot{M}(t) \leq \lambda$, or equivalently $M(t) \leq \lambda t+M(0)$ for $t>0$. The latter implies that the solution $u(x, t)$ exists for all times $t>0$ but it is unbounded, since the corresponding steady problem has no solutions.

For the case of general increasing $f$ and for some type of boundary conditions, the global existence of solution $u$ is proved, by using an eigenfunction expansion method, see [9].

\section{Conclusions}

We have studied the behaviour of the non-local parabolic equation

$$
u_{t}=u_{x x}-u_{x}+\frac{\lambda f(u)}{\left(\int_{0}^{1} f(u) d x\right)^{2}}
$$

with certain initial and boundary conditions where $f$ is the Heaviside function. In the case where $f(u)=H(1-u)$, so $f(u)$ is decreasing, comparison techniques can be applied. Two problems with different types of boundary conditions are studied. In both problems there exist $\lambda_{*}$ and $\lambda^{*}$ such that for $\lambda>\lambda^{*}$ or for $0<\lambda_{*}<\lambda<\lambda^{*}$ and sufficiently "warm" initial conditions the solution "blows up" in the sense that it becomes 1 at a finite time except for the points assigned zero boundary conditions. Regarding the original physical problem, this means that the food (or the substance undergoing the heating) loses all resistivity at temperature $u=1$, that is the heating ceases across the channel after finite time.

Acknowledgements: the authors thank the referees for several fruitful remarks. 


\section{References}

[1] D. G. Aronson, On the Green's function for second order parabolic equations with discontinuous coefficients, Bull. Amer. Math. Soc. 69 (1963), 841-847. E120

[2] C. H. Biss, S. A. Coombes and P. J. Skudder, The development applications of Ohmic heating for continuous processing of particulate foodstuffs in Process Engineering in the Food Industry, ed. R. W. Field, J. A. Howell, Elsevier, London, (1989), 17-27. E116

[3] X. Chen and A. Friedman, The thermistor problem for conductivity which vanishes at large temperature, Quart. Appl. Math. LI (1993), 101-115. E117

[4] X. Chen and A. Friedman, The thermistor problem with one-zero conductivity I, IMA Preprint Series No. 793 (1991). E117

[5] X. Chen, A. Friedman and B. Hu, The thermistor problem with one-zero conductivity II, IMA Preprint Series No. 873 (1991). E117

[6] M. Chiphot and B. Lovat, Some Remarks on non-local elliptic and parabolic problems, Nonlinear Anal. TMA 30, No. 7, (1997), 4619-4627. E127

[7] A. A. P. De Alwis and P. J. Fryer, Operability of the Ohmic heating process: electrical conductivity effects, J. Food Eng., 15 (1992), 21-48. E116

[8] P. J. Fryer, A. A. P. De Alwis, E. Koury, A. G. F. Stapley and L. Zang, Ohmic processing of solid-liquid mixtures: heat 
generation and convection effects, J. Food Eng. 18 (1993), 101-125. E116

[9] N. I. Kavallaris and D. E. Tzanetis, Blow-up and stability of a nonlocal diffusion-convection problem arising in Ohmic heating of foods, Diff. Integ. Eqns 15, (2002), No 3, 271-288. E116, E117, E118, E139

[10] A. A. Lacey, Thermal runaway in a non-local problem modelling Ohmic heating. Part I: Model derivation and some special cases, Euro. J. Appl. Math. 6 (1995), 127-144. E117, E118

[11] A. A. Lacey, Thermal runaway in a non-local problem modelling Ohmic heating. Part II: General proof of blow-up and asymptotics of runaway, Euro. J. Appl. Math. 6 (1995), 201-224. E118, E119, E120

[12] A. A. Lacey, D. E. Tzanetis and P. M. Vlamos, Behaviour of a non-local reactive convective problem modelling Ohmic heating of foods, Quart. J. Mech. Appl. Math, 52(4) (1999), 623-644. E116, E119

[13] O. A. Ladyzenskaja, V. A. Solonnikov and N. N. Ural'ceva, Linear and Quasilinear Equations of Parabolic Type, Transl. Math. Monog. Vol. 23, Amer. Math. Soc., Providence, R. I., (1968). E120

[14] C. P. Please, D. W. Schwendeman and P. S. Hagan, Ohmic heating of foods during aseptic processing, IMA J. Math. Bus. Ind., 5 (1994), 283-301. E116

[15] P. Skudder and S. Biss, Aseptic processing of food products using Ohmic heating, The Chemical Engineer, 2 (1987), 26-28. E116 
[16] I. Stakgold, Boundary Value Problems of Mathematical Physics, Vol. I, The Macmillan Company, Collier-Macmillan, London, (1970). E117

[17] R. Stirling, Ohmic heating - a new process for the food industry, Power Eng. J., 6 (1987), 365. E116

[18] D. E. Tzanetis, Blow-up of radially symmetric solutions of a non-local problem modelling Ohmic heating, Elec. J. Diff. Eqns., Vol. 2002, No. 11, (2002), 1-26. E118

[19] D. E. Tzanetis and P. M. Vlamos, Some interesting special cases of a nonlocal problem modelling Ohmic heating with variable thermal conductivity, Proc. Edinb. Math. Soc. (2) 44 (2001), No. 3, 585-595. E117, E118

[20] L. Zhang and P. J. Fryer, Models for the electrical heating of solid-liquid food mixtures, Chem. Eng. Sci., 48 (1993), 633-642. E116 